\title{
The resilience of the logarithmic law to pressure gradients: evidence from direct numerical simulation
}

\author{
RODERICK JOHNSTONE ${ }^{1} \dagger$, GAR Y N. COLEMAN \\ AND PHILIPPE R. SPALAR T \\ ${ }^{1}$ School of Engineering Sciences, University of Southampton, SO17 1BJ, UK \\ ${ }^{2}$ Boeing Commercial Airplanes, PO Box 3707, Seattle, WA 98124, USA
}

(Received 30 March 2009; revised 16 September 2009; accepted 18 September 2009; first published online 1 December 2009)

Wall-bounded turbulence in pressure gradients is studied using direct numerical simulation (DNS) of a Couette-Poiseuille flow. The motivation is to include adverse pressure gradients, to complement the favourable ones present in the well-studied Poiseuille flow, and the central question is how the scaling laws react to a gradient in the total shear stress or equivalently to a pressure gradient. In the case considered here, the ratio of local stress to wall stress, namely $\tau^{+}$, ranges from roughly $2 / 3$ to $3 / 2$ in the 'wall region'. By this we mean the layer believed not to be influenced by the opposite wall and therefore open to simple, universal behaviour. The normalized pressure gradients $p^{+} \equiv \mathrm{d} \tau^{+} / \mathrm{d} y^{+}$at the two walls are -0.00057 and +0.0037 . The outcome is in broad agreement with the findings of Galbraith, Sjolander \& Head (Aeronaut. Quart. vol. 27, 1977, pp. 229-242) relating to boundary layers (based on measured profiles): the logarithmic velocity profile is much more resilient than two other, equally plausible assumptions, namely universality of the mixing length $\ell=\kappa y$ and that of the eddy viscosity $v_{t}=u_{\tau} \kappa y$. In pressure gradients, with $\tau^{+} \neq 1$, these three come into conflict, and our primary purpose is to compare them. We consider that the Kármán constant $\kappa$ is unique but allow a range from 0.38 to 0.41 , consistent with the current debates. It makes a minor difference in the interpretation. This finding of resilience appears new as a DNS result and is free of the experimental uncertainty over skin friction. It is not as distinct in the (rather strong) adverse gradient as it is in the favourable one; for instance the velocity $U^{+}$at $y^{+}=50$ is lower by $3 \%$ on the adverse gradient side. A plausible cause is that the wall shear stress is small and somewhat overwhelmed by the stress and kinetic energy in the bulk of the flow. The potential of a correction to the 'law of the wall' based purely on $p^{+}$is examined, with mixed results. We view the preference for the log law as somewhat counter-intuitive in that the scaling law is non-local but also as becoming established and as highly relevant to turbulence modelling.

Key words: modelling, simulation, turbulent flows

\section{Introduction}

The cornerstone of our knowledge of wall-bounded turbulent flows, which we would describe as 'semi-theoretical', is the logarithmic law. The purest argument in its favour is that this layer is dominated by the shear stress, equal to $u_{\tau}^{2}$ (the density is 
set to 1 , and $u_{\tau}$ is the friction velocity). It is also dominated by the blocking effect of the wall, so that the wall distance $y$ sets the size of the largest, and stress-producing, eddies. The rest is dimensional analysis. (An excellent source is Bradshaw \& Huang 1995, who have also discussed the problem at hand here, on p. 173 of their work. Based on their position then, we expect that they will not be surprised by the present findings.)

Strictly, this reasoning applies only to a constant-stress layer: $u_{\tau}$ is a wall quantity but gives the shear stress across the entire layer in which universal behaviour is expected. Thus, $\tau(y)=u_{\tau}^{2}$. However, there is keen interest in layers with non-uniform stress, for two reasons. The first, which is practical, is the need to predict such flows via turbulence modelling; these are flows with pressure gradients and are of great importance. The second is that logarithmic behaviour is observed even in flows with favourable gradients (so that $\tau(y)<u_{\tau}^{2}$, often to a ratio of the order of $2 / 3$ or less). Examples include Poiseuille flows in channels and pipes and the Ekman layer (Spalart, Coleman \& Johnstone 2009). This poses a theoretical challenge of great interest, for the following reason.

Consider the flow outside the viscous-influenced layer. The log law can be motivated directly in terms of the velocity profile. If the shear flow is controlled by $u_{\tau}$ and $y$, dimensional analysis dictates the following for the shear rate:

$$
\frac{\mathrm{d} U}{\mathrm{~d} y}=\frac{u_{\tau}}{\kappa y},
$$

where $\kappa$ is the Kármán constant. The integral of (1) is the log law. Another approach to wall-bounded turbulence, attributed to Clauser, is through the eddy viscosity $v_{t}$. The shear stress is $-\overline{u^{\prime} v^{\prime}}=v_{t} \mathrm{~d} U / \mathrm{d} y$. Again by dimensional analysis,

$$
\frac{-\overline{u^{\prime} v^{\prime}}}{\mathrm{d} U / \mathrm{d} y} \equiv v_{t}=u_{\tau} \kappa y .
$$

Finally, Prandtl's mixing length equation is $v_{t}=\ell^{2} \mathrm{~d} U / \mathrm{d} y$, and by dimensional analysis,

$$
\frac{\sqrt{-\overline{u^{\prime} v^{\prime}}}}{\mathrm{d} U / \mathrm{d} y} \equiv \ell=\kappa y .
$$

In a constant-stress layer, outside the viscous layer, (1)-(3) are equivalent, since $-\overline{u^{\prime} v^{\prime}}=\tau(y)=u_{\tau}^{2}$.

Although (2) and (3) make use of the concepts of mixing length and eddy viscosity, they should not necessarily be viewed as 'turbulence modelling' while, in contrast, regarding (1) as 'theoretical'. All three amount to assertions about a length scale being proportional to the wall distance $y$, with $u_{\tau}$ setting the velocity scale. Note also that (3) has a slightly different nature, in that it does not involve the wall quantity $u_{\tau}$; it is local in $y$. This may explain its prominence in algebraic turbulence models, without indicating whether the primary reason is physics or convenience. In any case, in our view, none of the three arguments is more rigorous than the other two.

When the shear stress is not constant, (1)-(3) conflict. In a planar flow which is homogeneous in $x$, as here, the momentum equation reduces to $\mathrm{d} \tau / \mathrm{d} y=\mathrm{d} p / \mathrm{d} x$, so that the relationship between the stress gradient in $y$ and the pressure gradient in $x$ is simple. Furthermore, these quantities do not depend on $y$; so $\tau(y)$ is linear. This conflict has of course been known for a long time, and the desire to determine which argument survives, if any, is highly justified. In a series of papers, Galbraith, Sjolander and Head forcefully argued that experimental evidence favours (1) (Galbraith \& Head 
1975; Head \& Galbraith 1975; Galbraith, Sjolander \& Head 1977). Direct numerical simulation (DNS) evidence has been in agreement with this, but in a very limited manner (Spalart \& Watmuff 1993), outside of Poiseuille flows of course. This open question is quite important especially as the mixing-length equation (3) has been so widely used, but the challenge to it does not seem to have been followed up, other than by Johnson \& Coakley (1990). Naturally, no experimental measurement is perfect, especially for skin friction, but we have not found discussions that argue against the conclusions of these three papers on those grounds.

The circular aspect of these papers that came out in the mid-1970s is noted here, as it was then: they used an assumed logarithmic velocity profile to fit the measurements and calculate derivatives and in the end concluded that the log law is approximately valid. However, the fits are used to calculate the shear stress and then the mixing length via the momentum equation, which is not direct and has a chance of exposing the log-law assumption if it fails grossly. Nevertheless, independent measurement of the skin friction and near-wall profiles would have been preferable. Modern techniques in this field may prompt a fresh attempt at clearing the issue.

Two of the experimental Couette-Poiseuille cases of El Telbany \& Reynolds (1980) bracket ours, in terms of wall-to-wall stress ratio, and are close in Reynolds number. Their figures quite strongly support the law of the wall for velocity (1), with the skin friction obtained by extrapolating the shear stress to the wall, rather than velocitygradient measurements near the walls. They do not cite Galbraith et al. (1977), although their purposes had much overlap. Remarkably, they give for $\kappa$ a range of roughly $0.38-0.41$, and so the support they provide does not include a determination of the value of $\kappa$ that would be considered precise by today's standards.

It now appears possible to study this question by DNS, without excessively low Reynolds numbers obscuring the findings. While a vast body of Poiseuille-flow results is available and consistent, we feel that cases with adverse pressure gradient (APG) are essential. Each Couette-Poiseuille flow provides an APG and a favourable pressure gradient (FPG). These flows form a two-parameter family, conveniently described with the Reynolds number $R e$ (based on full channel width $2 h$ and the wall-velocity difference $\Delta U$ ) and with the ratio of shear stress at the two walls, that is to say the ratio of the stress at the APG wall to that at the FPG wall, by convention made smaller than 1 . In this study, we set $R e$ to 40000 and the stress ratio to 0.287 . These provide $y^{+}$ranges which, while not record setting, are far above the minimum needed to sustain turbulence.

A didactic reason to seek APG cases is the following: (1) and (2) are nonlocal, which creates the need to explain why the wall value of stress, through $u_{\tau}$, should dominate in a region in which $\tau$ is different. In FPG flows, the stress is weaker away from the wall, which could explain why the region closer to the wall dictates the velocity scale. Turbulent kinetic energy from below would be permeating the log layer. The weakness in this argument is that the eddy sizes are larger in the outer region and that the energy cascade is normally from large to small eddies. Furthermore, the turbulent diffusion of kinetic energy is very weak in the log layer, relative to production and dissipation. Sweeping statements are better avoided here.

In general, the Poiseuille-flow DNS has agreed with (1) fairly well (Hoyas \& Jiménez 2006), as has the Ekman-layer DNS (Spalart et al. 2009). Recall that the latter flow also has a favourable gradient, to the point that logarithmic behaviour is still observed fairly closely when the shear stress has already fallen by roughly $30 \%$. This is enough to bring out the conflict between (1)-(3), in the direction $\tau^{+}<1$. 


\begin{tabular}{|c|c|c|c|c|c|c|c|c|c|}
\hline Wall & $u_{\tau} / \Delta U$ & $p^{+}$ & $\Lambda_{x}^{+}$ & $\Lambda_{z}^{+}$ & $\Delta x^{+}$ & $\Delta z^{+}$ & $y_{10}^{+}$ & $\Delta y_{c}^{+}$ & $\Delta t^{+}$ \\
\hline APG & 0.0168 & 0.0037 & 4217 & 2108 & 5.9 & 2.4 & 2.3 & 4.4 & 0.033 \\
\hline FPG & 0.0313 & -0.00057 & 7877 & 3939 & 10.9 & 4.6 & 4.3 & 8.2 & 0.12 \\
\hline
\end{tabular}

With adverse gradient, the shear stress is relatively weak at the wall, raising the possibility that its influence on the region that is a candidate for universal behaviour would also be weakened. In any case, the simulation task here is to produce flows with appreciable deviations of $\tau(y)$ from $u_{\tau}^{2}$, in both directions, and to test the three candidate length scales. Possible outcomes include a clear 'win' for one of the three or a trend towards an intermediate behaviour. Unclear trends, or conflicting trends between the two walls, could of course not be ruled out. Note that (3) will fall between (1) and (2), in terms of deviations from universal behaviour, because

$$
\left(\frac{\ell}{\kappa y}\right)=\sqrt{\left(\frac{u_{\tau}}{\kappa y \mathrm{~d} U / \mathrm{d} y}\right)\left(\frac{v_{t}}{\kappa y u_{\tau}}\right)} .
$$

In a constant-stress layer, this amounts to $1=\sqrt{1 \times 1}$. Note also that for the present linear variations of $\tau(y)$ - which are typical of flows with moderate pressure gradients for which one or another universal behaviour might be found - the classic framework assumed here will not admit the outcome that two of the properties are both satisfied but with a different value of $\kappa$. (This work certainly does not involve a search for 'adaptive' $\kappa$ values.) We thus expect that one candidate will succeed, at most.

\section{Numerical considerations}

The simulation is performed using a version of the Fourier/Chebyshev spectral channel code of Kim, Moin \& Moser (1987), from which it differs algorithmically in the time integration: a third-order Runge-Kutta/Crank-Nicolson scheme is used here. Moving-wall boundary conditions have also been added, and the reference frame for time integration is at the average velocity of the two walls.

The computational domain is of size $4 \pi h \times 2 h \times 2 \pi h$, in $x, y, z$ respectively, with a grid resolution (in real space) of $720 \times 241 \times 864$ (the $3 / 2$ rule is applied in $x$ and $z$ for dealiasing). The corresponding resolution is given in wall units in table $1\left(y_{10}^{+}\right.$is the height of the 10th wall-normal grid point and $\Delta y_{c}^{+}$is the centreline resolution). These numbers improve on or match those found adequate by Kim et al. (1987) in their channel. (The Runge-Kutta scheme is also a slight improvement on their Adams-Bashforth scheme.) The channel-flow DNS and pipe flow from other codes with comparable resolutions show good agreement at similar pressure gradients (cf. figure 2 and the internal flows near the origin of figure 6), typically within 0.1 unit in $U^{+}$, which is sufficient for present purposes.

Regarding the streamwise and lateral domain sizes $\Lambda_{x}$ and $\Lambda_{z}$, Lee \& $\operatorname{Kim}(1991)$ concluded, after extensive DNS studies of Couette flow in larger domains, that $4 \pi h \times(8 \pi / 3) h$ is optimal. Our domain width, the one that is time honoured in Poiseuille flow, is $3 / 4$ of theirs; its effect is discussed in $\S 3.4$. Pure Couette flow simulations (not discussed in detail here) have also been carried out by the authors at $R e_{\tau}$ comparable to that of the FPG side of the Couette-Poiseuille simulation, once with the same grid and domain as above and once with a double-length domain (and the same resolution). These indicated that the smaller domain size is less than ideal 


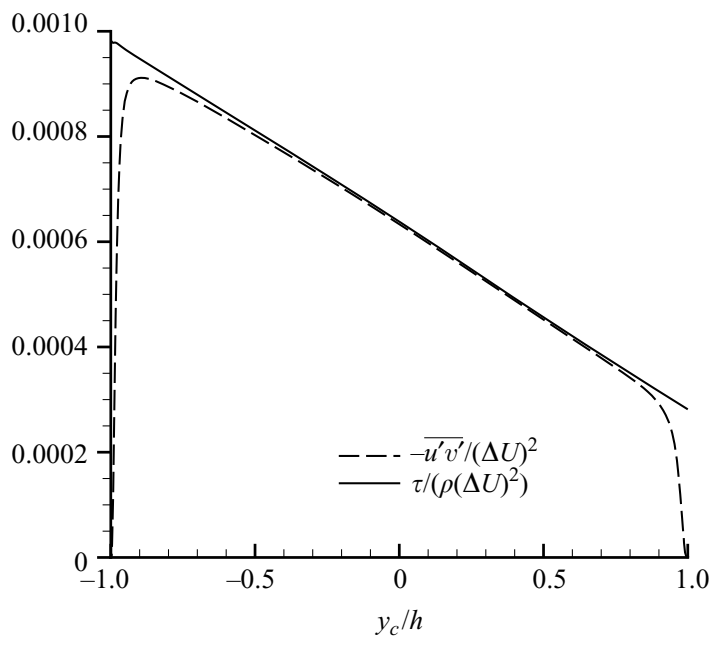

FIGURE 1. Shear stress.

for Couette flow; the $x$-direction two-point correlation $R_{u и}$ remains above 0.56 on the centreline. This measure is below 0.36 in the Couette-Poiseuille simulation discussed here (and 0.32 for the double-length Couette flow). The lengths defined by (1)-(3) were not found to be very sensitive to domain size in Couette flow, certainly for $y^{+}<400$.

\section{Results}

\subsection{General presentation}

Figure 1 shows the Reynolds and total shear stresses across the channel $\left(y_{c}\right.$ is the distance from the centreline), to exhibit the extent of the variation from one wall to the other and to outline the thickness of the viscous-influenced layers. This will serve as a reference for later figures. The skin-friction ratio of about 0.3 is the major design parameter. The left wall will be referred to as 'FPG' and the right one as 'APG'. The FPG wall is, at first sight, very similar to a Poiseuille wall, but the shear stress does not reach zero before the influence of the opposite wall is felt. As a result, attempting to define an equivalent Poiseuille Reynolds number $R e_{\tau} \equiv-1 / p^{+}$would not be appropriate. The APG wall layer is more distinctive. The total stress reaches about twice the wall value before the opposite wall is felt, based on intuition and on the findings of $\S 3.2$. Thus, the pressure gradient is strong, which seemed a sensible choice for this first case. In wall units, the pressure gradient is +0.0037 , compared with -0.00057 for FPG. At $y^{+}=100$, where universal behaviour might be found, the two walls have $\tau^{+}=1.37$ and 0.943 , respectively.

In figure 2(a), the velocity profile is shown with the same axes as in figure 1, displaying a modified Couette-flow shape, with a shift towards the velocity of the APG wall as could be expected from the smaller friction velocity there. The inflection point may serve as a notional border between 'zones of influence'.

The velocity profiles now in wall units and logarithmic $y$ axis in figure 2(b) reveal a 'visual' $\log$ layer to $y^{+}$of about 250 on the FPG side (where the shear stress has fallen by $14 \%$ ) and very close agreement with Poiseuille results by Hoyas \& Jiménez (2006). Its approximate Kármán constant, simply using a ruler, is 0.42 . However, the Kármán measure $\mathrm{d}\left(\log y^{+}\right) / \mathrm{d} U^{+}$(not shown), used in our flat-plate and Ekman-layer studies, does not remain constant to \pm 0.01 over any appreciable range of $y^{+}$. On the APG side, the visual log layer is much shorter, barely to 120 , if it exists at all; many 


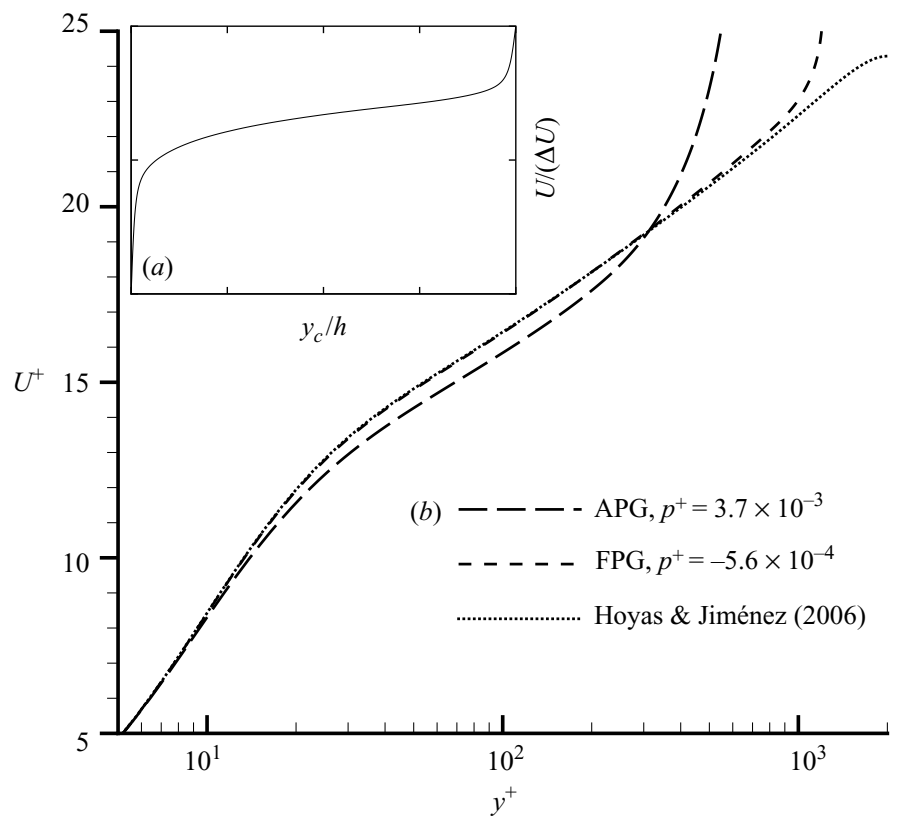

FIGURE 2. Velocity profiles in $(a)$ the outer layer and $(b)$ wall units. The plane Poiseuille results of Hoyas \& Jiménez (2006) are included for comparison $\left(p^{+}=-5.0 \times 10^{-4}\right)$.
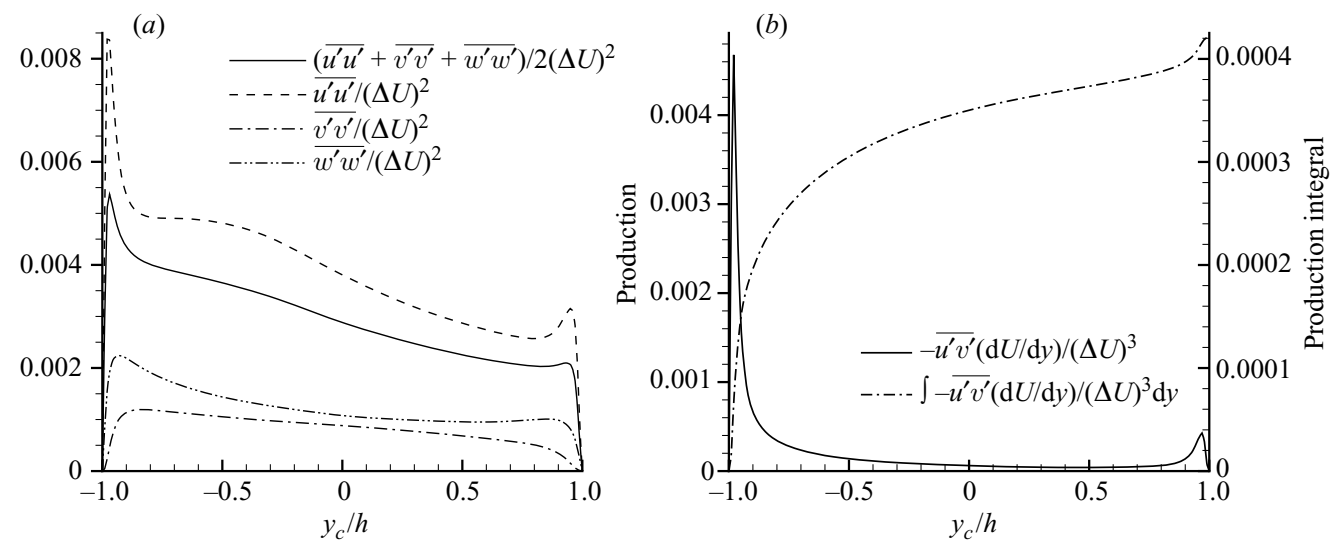

Figure 3. (a) Normal Reynolds stresses and turbulent kinetic energy. (b) Production and its integral. The wall shear stress is 0.00098 and 0.00028 at the FPG and APG walls, respectively.

observers will only see an inflection point with a slope about $1 / 0.45$. In addition, this part of the curve is decidedly lower than the standard law, the one that applies over a wide range of weaker pressure gradients, making it highly suspect as a log layer. This lower reach in $y^{+}$is partly due to the lower friction velocity, which lowers $y^{+}$for the same $y / h$, but as mentioned earlier the pressure gradient is also about six times larger in wall units. The differences in this figure are examined again in $\S 3.3$.

Figure 3 $(a)$ contains the normal Reynolds stresses, revealing moderately different levels in FPG and APG (wall units not used here). The reduction of the near-wall peak on the APG side is a result of both lower effective Reynolds number and lower energy relative to the centre region. Figure $3(b)$ shows the production of turbulent 


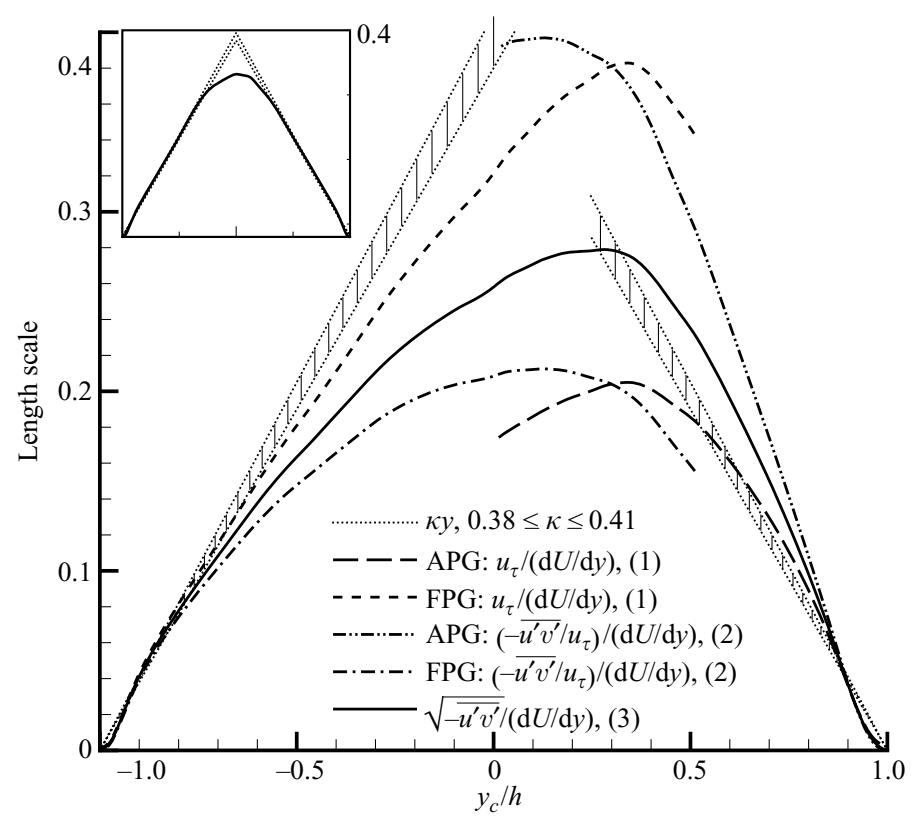

FIGURE 4. Turbulence length scales. The inset shows results for pure Couette flow (for which (1)-(3) are essentially coincident).

kinetic energy, again with an eye towards determining zones of influence. The peak value at the FPG wall is over 10 times larger than that at the APG wall, following as expected the fourth power of $u_{\tau}\left(0.3^{2}\right.$ is 0.09$)$. The amount of energy production plausibly attributed to each of the layers via its integral versus $y$ is in a ratio closer to six, which still corroborates the dominance of the FPG wall.

\subsection{Test of the scaling laws}

Figure 4(a) displays a direct test of (1)-(3), providing the answer to the central question of this study. The three quantities, each of which equals $\kappa y$ in the ideal situation, are shown. The viscous buffer layers are thin enough not to invade the regions of interest. The curves for (1) and (2) are discontinuous by a factor $\sqrt{0.3}$ because they involve the friction velocity of the wall they are associated with. Some overlap has been allowed, bracketing $y=0.25$, which appears to separate the zones of influence of the two walls. The inset in figure 4 shows the result of a pure Couette flow DNS with $R e_{\tau} \approx 600$. Except very near the wall, (1)-(3) collapse, and between $y^{+}=100$ and $\left|y_{c} / h\right|>0.3$ they are consistent with $\ell=\kappa y$. Figure 5 additionally shows the length scales derived from (1)-(3) in wall units, to illustrate the degree to which they exhibit near-wall universality.

The indications in figures 4 and 5 are strong: (1) is much closer to being satisfied than (3), which in turn is closer to being satisfied than (2). Yet even on the FPG side, (1) is not precisely satisfied; the implied $\kappa$ peaks near 0.43 just above the buffer layer, falling to 0.38 at about $y^{+}=200$ and gradually dropping to 0.32 at the centreline. The peak and approach to a universal $\kappa$ from above are, very probably, part of the ideal law of the wall, as indicated by the fit of Chauhan, Nagib \& Monkewitz (2007). On the APG side, it may again be debated whether a convincing log layer exists; if 


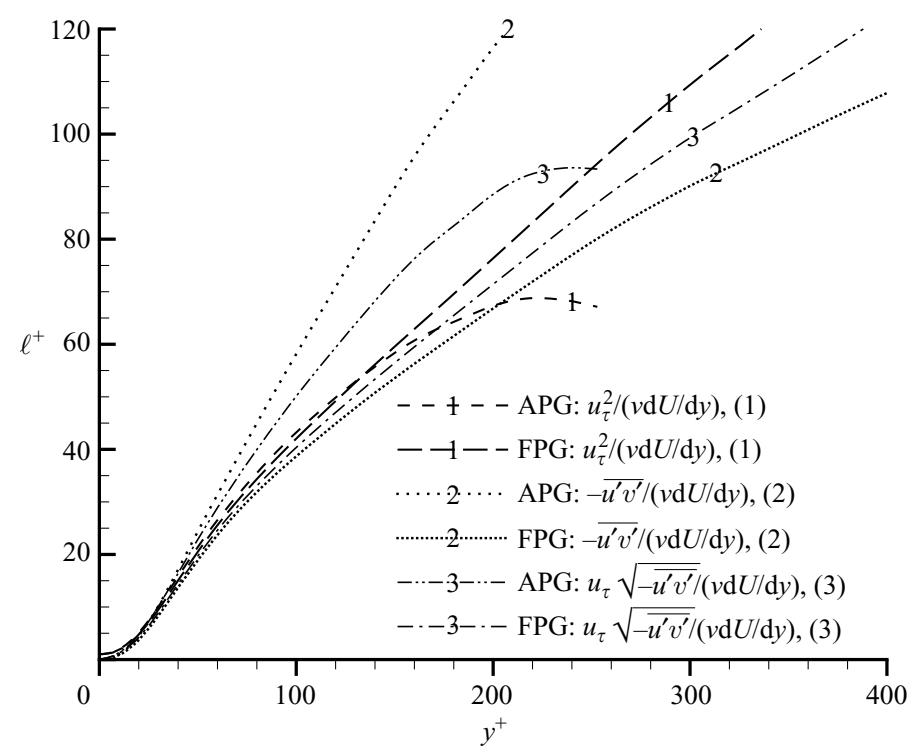

FIGURE 5. Turbulence length scales in wall units.

it does, it requires a higher $\kappa$, which we do not consider acceptable on theoretical grounds. However, (1) is clearly a much better approximation than (3) and of course (2) (recall (4)); near-linear behaviour applies roughly up to where the normalized stress $\tau^{+}$has increased to $3 / 2$, i.e. where the deviation from an ideal constant-stress layer is strong. The result is not trivial.

Figure 5 confirms the ranking between the equations, in that (2) and (3) rapidly fan out in response to pressure gradients. They fail comprehensively in the framework of a law of the wall (for their respective quantities), which implies a unique profile. To be impartial, we note these two figures would not definitely defeat the proposal of using (2) or (3) with an adjustment of $\kappa$ for each wall. Proposal (3) would require $\kappa>0.6$ in APG and $\kappa<0.3$ for FPG; in the case of (2) somewhat more moderate values could be used (perhaps 0.5 and slightly over 0.3 ). Both would require virtual origins. However, these changes can hardly be conceived as 'adjustments'. Furthermore, they would be very unlikely to apply close to the wall at high Reynolds number, since that region would have $\tau^{+}$close to 1 and $p^{+}$much smaller than here, and we would strongly expect $\kappa$ to return to typical values there, rather than values such as 0.3 or 0.6 . Thus the recovery of (2) or (3) via a variable $\kappa$ is very problematic, far more so than the attitude that (1) is 'resilient' without being exact. The local character of the mixing-length formula would also be lost if $p^{+}$dependence were to be introduced.

\subsection{Pressure-gradient correction to the law of the wall}

The downward shift of the APG profile in figure $2(b)$ evokes earlier results, both experimental and from DNS (Nagano, Tagawa \& Tsuji 1992; Spalart \& Watmuff 1993). Seeing such results and having now noted that the velocity law of the wall is favoured over the other two scaling laws, it is tempting to conjecture the existence a 'pressure-gradient law of the wall' in which $U^{+}$is a function of $y^{+}$and $p^{+}$, say, instead of only $y^{+}$. The word 'adjustment' would be justified here. Such conjectures have been 


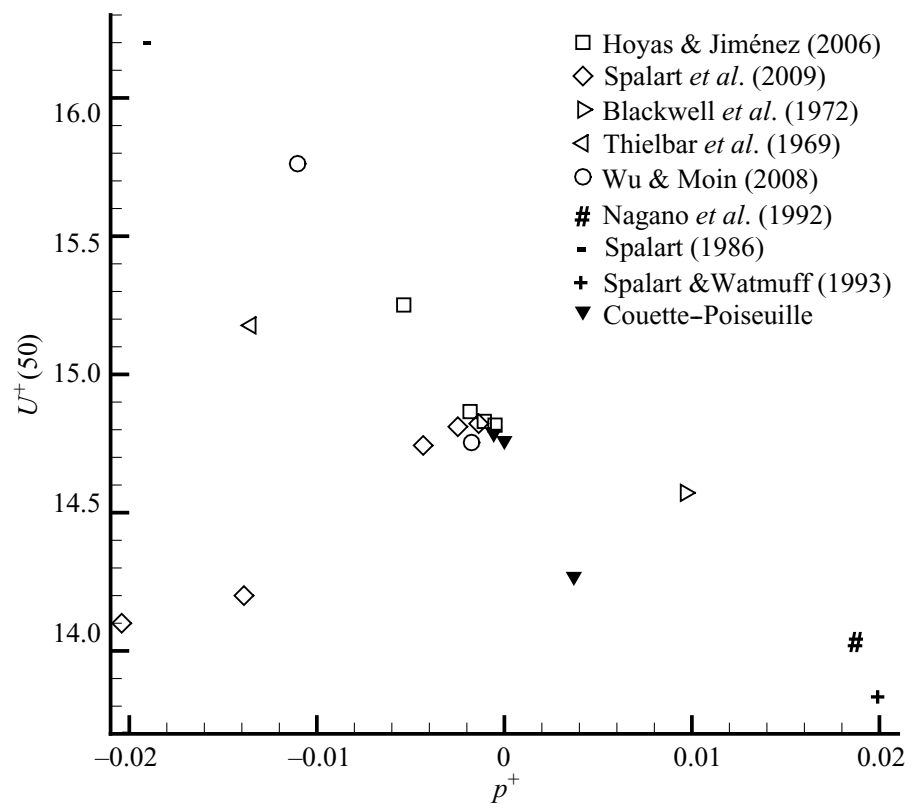

FIGURE 6. Plot of $U^{+}$at $y^{+}=50$, from simulations and experiment. Results of Blackwell et al. (1972) and Thielbar, Kays \& Moffatt (1969) adjusted as in Volino \& Simon (1997).

discussed in the literature, usually to improve wall functions and derived from mixinglength assumptions (Volino \& Simon 1997), without being widely accepted. There has been too little systematic comparison with data, and there are conceptual hurdles linked to the non-uniqueness of the measure of the pressure gradient or the stress gradient. Using $p^{+}$rather than $\mathrm{d} \tau^{+} / \mathrm{d} y_{y=0}^{+}$is arbitrary; these two quantities are equal at plane walls but differ by a factor of 2 in the pipe. In other flows such as sink flows and Ekman layers, the $y$ dependence of $\tau$ is not linear, and a function of $\left(y^{+},\left(\tau^{+}-1\right) / y^{+}\right)$would be as justified as a function of $\left(y^{+}, p^{+}\right)$.

Stimulated by two reviews, the $p^{+}$conjecture is given a test at face value in figure 6 , which shows $U^{+}$at $y^{+}=50$ versus $p^{+}$for a range of flows, from experiments and DNS (Spalart 1986; Nagano et al. 1992; Spalart \& Watmuff 1993; Volino \& Simon 1997; Hoyas \& Jiménez 2006; Spalart et al. 2009; Wu \& Moin 2008). The same test applied at $y^{+}=1$, limited to DNS, agrees very well with the viscous Taylor expansion $\partial U^{+} / \partial p^{+}=(1 / 2) y^{+2}$, neglecting the Reynolds shear stress. Note that using $\mathrm{d} \tau^{+} / \mathrm{d} y^{+}$ instead of $p^{+}$would move the pipe-flow point of $\mathrm{Wu} \&$ Moin to the right, half-way to the origin, placing it well outside the band of channel results. Using $\left(\tau^{+}-1\right) / y^{+}$ would move all the boundary-layer results towards the origin $\left(p^{+}=0\right)$.

The results confirm a general tendency for $U^{+}(50)$ to decrease in increasingly adverse gradients (whereas $U^{+}(1)$ increases), except in the Ekman layer which shows the opposite trend. Deciding on the conjecture that $U^{+}$at fixed $y^{+}$is a unique function of $p^{+}$, based on this figure, is a matter of judgement. If scatter of the order of \pm 0.4 in $U^{+}$is accepted and the Ekman results are for some reason ignored, the conjecture might be correct. Poiseuille results agree with the conjecture, but trivially so, since dimensional analysis dictates for $U^{+}$a function of $\left(y^{+}, R e_{\tau}\right)$ and $p^{+}=1 / R e_{\tau}$. The difference between the Poiseuille and pipe DNSs at $p^{+} \approx-0.0018$, namely about 0.1 in $U^{+}$, can probably be viewed as scatter (because of grid, discretization or time sample). The Couette and Couette-Poiseuille results are consistent with the Poiseuille 
trend, and non-trivially so. The channel and pipe results are not far from a line $U^{+}(50)=17.75-105 p^{+}$(although the pipe result would be well above this line if $\mathrm{d} \tau^{+} / \mathrm{d} y^{+}$were used rather than $p^{+}$). Older experimental results show less slope, but this is precisely what would be expected from indirect methods of evaluating skin friction.

The Ekman-layer results strongly disagree with the idea of a unique curve (Spalart et al. 2009). We do note that they were also generated by us, which could raise the question of a bias; however, another code was used. Other measures such as $\left(\tau^{+}-1\right) / y^{+}$would not lessen this conflict, and the Ekman three-dimensional effects at this wall distance are very weak. Results from a boundary layer with zero pressure gradients would line up vertically at $p^{+}=0$, with that for $R_{\delta^{*}}=500$ about 0.5 units higher than that for $R_{\delta^{*}}=2000$ (Spalart 1988). This would make them protrude strongly upwards, but using $\left(\tau^{+}-1\right) / y^{+}$would this time move these points left and would reduce the discrepancy. Thus, different interpretations can be made. In addition, at the Reynolds numbers of many of the flows used for figure 6 especially for $p^{+}<-0.005$, it is far from obvious that the compromise height we have chosen, namely $y^{+}=50$, is not already somewhat into the outer region of the flow. Therefore, using these points in a quest for a 'law of the wall' is questionable.

Faced with the Ekman-layer results, some analysts will argue that all channel flows obey the same law, while pipes and especially boundary layers each have their own. We believe this runs against all classical arguments given in favour of a law of the wall, but this must be regarded as an open question at present. While recording our position that the idea of a $p^{+}$correction is probably unsuccessful, we leave this point, which is finer than the one which motivated our study, for future debates.

\subsection{Narrow domain}

This test was also suggested by a reviewer; its purpose is to separate the effect of the opposite wall from that of the nearest wall, by introducing a third length scale which is more intrusive than the opposite wall. In particular, this should suppress the largest turbulent structures responsible for communication between the walls, which are expected to have transverse dimensions comparable to the channel height $2 h$ (Lee \& Kim 1991). The simulation was repeated with the same parameters, except with a lateral period of $\pi h / 2$ rather than $2 \pi h$, expecting this to be sufficient to remove the largest turbulent structures. (The reviewer ventured that the turbulence may not even survive but had suggested an extremely narrow domain of $h / 2$; we compromised.)

Results indeed reveal a very strong effect (figures 7 and 8); the mixing length now has a plateau near $0.13 \mathrm{~h}$, very plausibly dictated by the confinement. Figure 7 is to the same scale as figure 4 to aid comparison. This is a favourable result in that the same plateau for the standard domain would logically be near $0.52 h$, but the mixing length in figure 4 does not exceed $0.28 \mathrm{~h}$. It follows that confinement of large-scale turbulence is by the other wall, not the lateral domain size, and the value of the latter given in table 1 is adequate for our purposes, which concern the length scales shown in the figure.

Figure 8 is also favourable in that the ranking between (1)-(3) remains the same as before. The only difference is that the agreement between curves associated with the FPG and APG walls does not extend as far as it did, and the region dominated by the nearest wall is better indicated, as was hoped for. 


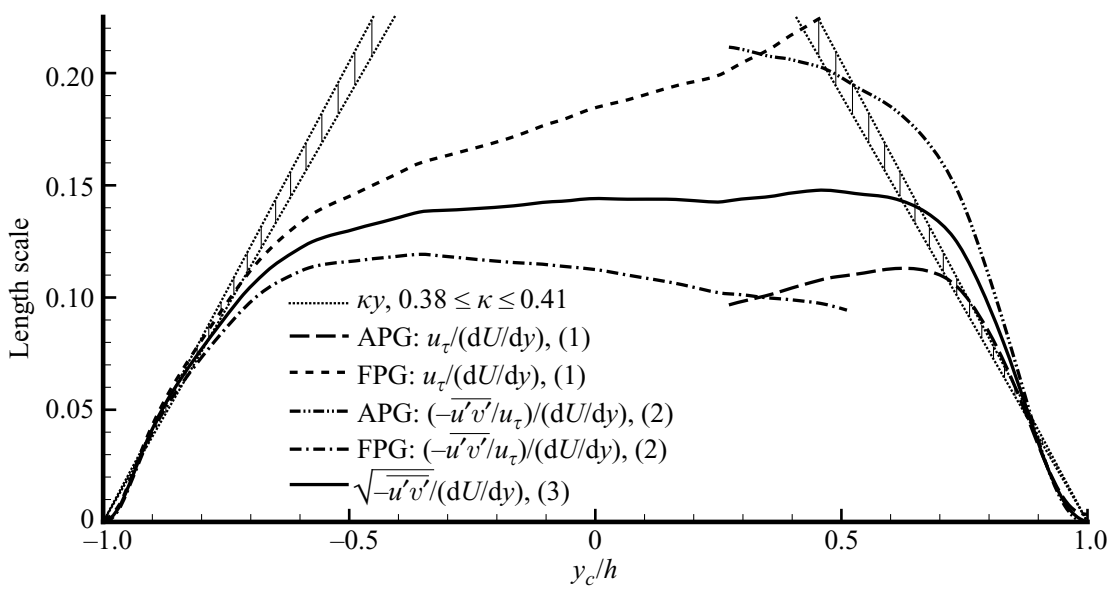

FiguRE 7. Turbulence length scales, with a narrow domain.

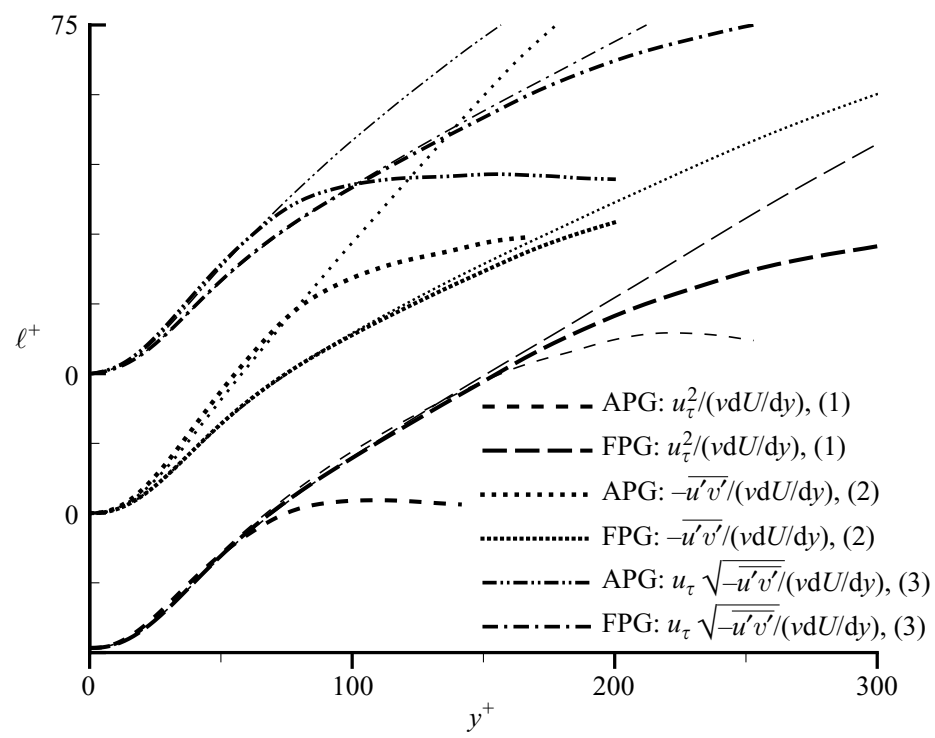

FIGURE 8. Turbulence length scales in wall units: thin lines represent narrow domain results, heavier lines those from the original domain.

\section{Conclusion}

This Couette-Poiseuille study points to a strong preference among the three hypotheses, one that is the same on the FPG and APG sides. This is only a preference, and there is no indication of any of the equations being exact or even accurate within a few per cent. The Reynolds number is marginal on the APG side, but a decisive increase will have to wait for more powerful computers. We believe that the present results, limited as they are, deserve some attention and that this short paper may initiate fruitful reflection and further experimental and DNS studies. The experimental benchmark used here is from 1980. The agreement is strong with, in our view, an under-appreciated set of mid-1970s experiments. Perhaps it is time to use modern measurement techniques to revisit these experiments. 
In terms of the prevalent 'semi-theory' of turbulence, the findings are arguably unfortunate, in that the most successful formula, namely (1), is not the one with the most intuitive appeal, at least to the authors. It is not local, and there is no doubt that it will break down in very strong APG. Millikan overlap arguments can be made in Couette-Poiseuille flow and will predict logarithmic behaviour, which we also expect DNS at higher Reynolds number would confirm. However they are asymptotic $\left(\tau^{+} \rightarrow 1, y / h \ll 1\right)$, when the interest here is in the regions in which $\tau^{+}$ is not very close to 1 , say where its values reach $2 / 3$, or $3 / 2$. In other words, the difficulty is to explain the manifest, if approximate, maintenance of the log law deep into the defect-law region, in the terminology of overlap arguments. The closeness of Poiseuille velocity profiles to the log law almost to the centreline was noticed long ago and was viewed as a coincidence, which the boundary layer does not share. This issue may not be truly a theoretical one, but its practical importance to turbulence modelling is paramount.

This work was sponsored by the Engineering and Physical Sciences Research Council through the UK Turbulence Consortium (Grant EP/D044073/1) and the Turbulence Platform Grant (GR/582947/01). Computations were made on the UK/EPSRC HECToR system.

\section{REFERENCES}

Blackwell, B. F., Kays, W. M. \& Moffat, R. J. 1972 The Turbulent Boundary Layer on a Porous Plate: An Experimental Study of the Heat Transfer Behaviour with Adverse Pressure Gradients. HMT-16, Thermosciences Division, Mechanical Engineering Department, Standford University.

Bradshaw, P. \& Huang, G. P. 1995 The law of the wall in turbulent flow. Proc. R. Soc. Lond. A 451, 165-188.

Chauhan, K., Nagib, H. M. \& Monkewitz, P. A. 2007 On the composite logarithmic profile in zero pressure gradient turbulent boundary layers. In 45th AIAA Aerospace Sciences and Meeting and Exhibit, 8-11 January 2007, Reno, Nevada. Also Paper No. 2007-0532. AIAA.

El Telbany, M. M. M. \& Reynolds, A. J. 1980 Velocity distributions in plane turbulent channel flows. J. Fluid Mech. 100, 1-29.

Galbraith, R. A. McD. \& Head, M. R. 1975 Eddy viscosity and mixing length from measured boundary layer developments. Aeronaut. Quart. 26, 133-154.

Galbraith, R. A. McD., Sjolander, S. \& Head, M. R. 1977 Mixing length in the wall region of turbulent boundary layers. Aeronaut. Quart. 27, 229-242.

Head, M. R. \& Galbraith, R. A. McD. 1975 Eddy viscosity and entrainment in equilibrium boundary layers. Aeronaut. Quart. 27, 229-242.

Hoyas, S. \& JimÉnEZ, J. 2006 Scaling of the velocity fluctuations in turbulent channels up to $R e_{\tau}=2003$. Phys. Fluids 18, 011702.

Johnson, D. A. \& COAKLEY, T. J. 1990 Improvements to a nonequilibrium algebraic turbulence model. AIAA. J. 28 (11), 2000-2003.

Kim, J., Moin, P. \& Moser, R. D. 1987 Turbulence statistics in a fully developed channel flow at low Reynolds number. J. Fluid Mech. 177, 133-166.

LEE, M. J. \& KIM, J. 1991 The structure of turbulence in simulated plane Couette flow. In Eighth Symposium on Turbulent Shear Flows, Technical University of Munich, Munich, Germany.

Nagano, Y., Tagawa, M. \& Tsuji, T. 1992 Effects of adverse pressure gradient on mean flows and turbulence statistics in a boundary layer. In Turbulent Shear Flows 8, (ed. F. Durst, B. E. Launder \& R. Friedrich). Springer.

SPAlarT, P. R. 1986 Numerical study of sink-flow boundary layers. J. Fluid Mech. 172, 307328.

SPALART, P. R. 1988 Direct simulation of a turbulent boundary layer up to $R_{\theta}=1410$. J. Fluid Mech. 187, 61-98. 
Spalart, P. R., Coleman, G. N. \& Johnstone, R. 2009 Retraction: direct numerical simulation of the Ekman layer. Phys. Fluids. To appear.

Spalart, P. R. \& Watmuff, J. H. 1993 Experimental and numerical study of a turbulent boundary layer with pressure gradients. J. Fluid Mech. 249, 337-371.

Thielbar, W. H., Kays, W. M. \& Moffat, R. J. 1969 The Turbulent Boundary Layer: Experimental Heat Transfer with Blowing, Suction, and Favorable Pressure Gradient. HMT5, Thermosciences Division, Mechanical Engineering Department, Stanford University.

Volino, R. J. \& Simon, T. W. 1997 Velocity and temperature profiles in turbulent boundary layers experiencing streamwise pressure gradients. J. Heat Transfer 119, 433-439.

Wu, X. \& MorN, P. 2008 A direct numerical simulation study on the mean velocity characteristics in turbulent pipe flow. J. Fluid Mech. 608, 81-112. 